\title{
Mecánica cuántica en las representaciones de coordenadas y momento a través tres problemas sencillos
}

\section{Quantum Mechanics in Coordinate and Moment Representations Through Three Simple Problems}

\author{
E. J. Bolaños ${ }^{1}$, D. Suescún ${ }^{2}$ y D. A. Rasero ${ }^{3}$
}

\section{Resumen}

En este trabajo presentamos la solución de la ecuación de Schrödinger estacionaria en la representación de coordenadas y de momento para tres problemas sencillos: el potencial lineal, el oscilador armónico y el potencial tipo delta de Dirac. Verificamos a través del cálculo explícito que las funciones de onda obtenidas en cada representación están conectadas a través de la transformada de Fourier, mostrando que ambas soluciones constituyen dos representaciones complementarias del mismo estado cuántico. Desde un punto de vista pedagógico esto muestra que ambas soluciones contienen información de igual valor teórico y constituyen dos representaciones complementarias.

Palabras clave: ecuación de Schrödinger, representación de coordenadas y de momento, transformada de Fourier

\begin{abstract}
In this paper we present the solution of the stationary Schrödinger equation in the coordinates and momentum representations for three simple problems: the linear potential, the harmonic oscillator and the Dirac delta potential. We verify through explicit calculation that the wave functions obtained in each representation are connected through the Fourier transform, showing that both solutions constitute two complementary representations of the same quantum state. From a pedagogical point of view, this shows that both solutions contain information of equal theoretical value and constitute two complementary representations.
\end{abstract}

Keywords: Schrödinger equation, coordinates and momentum representations, Fourier transform

Recepción: 18 -ago-2020

Aceptación: 10-dic-2020

\footnotetext{
${ }^{1}$ Estudiante del Programa de Física, Universidad Surcolombiana, Dpto. de Ciencias Naturales, Grupo de Física Aplicada FIASUR, Neiva, Colombia. Correo electrónico: johana9369@gmail . com

${ }^{2}$ Universidad Surcolombiana, Dpto. de Ciencias Naturales, Grupo de Física Aplicada FIASUR, Neiva, Colombia.

Correo electrónico: daniel. suescun@usco.edu.co

${ }^{3}$ Universidad Surcolombiana, Dpto. de Ciencias Naturales, Grupo de Física Aplicada FIASUR, Neiva, Colombia.

Correo electrónico: diego.rasero@usco.edu.co
} 


\section{Introducción}

En mecánica cuántica es posible abordar la mayoría de problemas a través de dos representaciones continuas: la representación de posición y la representación de momento. Sin embargo, es común que en los libros de textos y cursos tradicionales de mecánica cuántica $[1,2,3,4,5]$ no se profundice en el estudio de la representación de momento lineal, suministrando únicamente una introducción formal y breve del tema. El hecho de que la ecuación de Schrödinger en la representación de momento se convierta en una ecuación integral, para la cual los estudiantes de pregrado pueden no tener las herramientas matemáticas suficientes para darle solución, en lugar de una típica ecuación diferencial como en la representación de coordenadas puede ser una de las razones por las que en los textos y cursos a nivel de pregrado se dedique mayor atención a esta última representación y se indique simplemente que la función de onda en el espacio de momento se calcula a través de la transformada de Fourier de la función en el espacio de coordenadas. Pero debe recalcarse, como lo señala Guillaumín-España et al. [6], que los operadores de posición y momento tienen roles simétricos en la teoría y por tanto, a nuestro parecer, el uso extendido de la representación de posición obedece a cuestiones pedagógicas.

Algunos autores han incursionado en la solución de problemas unidimensionales en la representación de momento. Por ejemplo, el potencial lineal, oscilador armónico, el átomo de Hidrógeno unidimensional y algunos pozos y barreras de potencial $[6,7,8,9]$ pero en la mayoría, no se presenta una versión unificada en la que se resuleva el problema en ambas representaciones y además se verifique que tales soluciones están conectadas por la transformada de Fourier.

Comenzamos en la Sección 2 revisando las propiedades básicas de las representaciones de coordenadas y de momento, introduciendo primero la ecuación de Schrödinger en la notación de Dirac y luego definiendo las bases de posición y momento con las cuales se construyen las funciones de onda en cada representación y se establece su conexión mediante la transformada de Fourier. En la sección 3 se resuelve la ecuación de Schrödinger estacionaria en las representaciones de coordenadas y de momento, cuando el operador energía potencial es una función lineal, cuadrática (oscilador armónico) y tipo delta de Dirac. Además, verificamos que las funciones de onda obtenidas en cada representación están conectadas a través de la transformada de Fourier. Esperamos que este trabajo contribuya a que los estudiantes de pregrado en física comprendan que ambas soluciones contienen información de igual valor teórico y constituyen dos representaciones complementarias del mismo estado cuántico.

\section{Teoría}

En mecánica cuántica, la evolución temporal del estado de un sistema $|\Phi(t)\rangle$ queda determinada por la ecuación de Schrödinger [1,2]

$$
i \hbar \frac{d}{d t}|\Phi(t)\rangle=\hat{\mathbb{H}}|\Phi(t)\rangle,
$$

donde $\hbar$ es la constante de Planck racionalizada y $\hat{\mathbb{H}}$ es el operador Hamiltoniano. A esta manera de analizar la dinámica del sistema se le conoce como imagen de Schrödinger de la mecánica cuántica.

El operador Hamiltoniano se puede construir en términos de los operadores posición $\hat{\mathbb{R}}$ y momento lineal $\hat{\mathbb{P}}$ como

$$
\hat{\mathbb{H}}(\hat{\mathbb{R}}, \hat{\mathbb{P}}, t)=\frac{\hat{\mathbb{P}}^{2}}{2 m}+\hat{\mathbb{V}}(\hat{\mathbb{R}}, t),
$$

donde $\hat{\mathbb{V}}(\hat{\mathbb{R}}, t)$ es el operador energía potencial.

Cuando $\hat{\mathbb{V}}$, y por tanto $\hat{\mathbb{H}}$, no depende del tiempo y el estado en el instante inicial $t=t_{0}$ es $\left|\Phi\left(t_{0}\right)\right\rangle$, la solución de (1) se puede escribir como

$$
|\Phi(t)\rangle=e^{-\frac{i}{\hbar} \hat{\mathbb{H}}\left(t-t_{0}\right)}\left|\Phi\left(t_{0}\right)\right\rangle .
$$

Además, el operador $\mathbb{H}$ satisfará la ecuación de valores y estados propios

$$
\hat{\mathbb{H}}\left|\psi_{n}\right\rangle=E_{n}\left|\psi_{n}\right\rangle
$$

en la que $n$ etiqueta cada valor y estado propio. La ecuación (4) es conocida con el nombre de ecuación de Schrödinger estacionaria o independiente del tiempo. Los valores propios de energía $E_{n}$ son reales y el conjunto de estados $\left\{\left|\psi_{n}\right\rangle\right\}$ constituye una base ortonormal y completa en la que expandimos $\left|\Phi\left(t_{0}\right)\right\rangle$ para encontrar $|\Phi(t)\rangle$, solución de (1). 
En este trabajo solucionamos la ecuación (4), en el caso unidimensional, considerando las dos representaciones continuas de posición y momento.

\subsection{Representaciones de Posición y Momento}

Si $\hat{\mathbb{X}}$ y $\hat{\mathbb{P}}_{x}$ son los operadores que representan aparatos de medida capaces de medir la posición y el momento lineal de una partícula en el eje $x$,

$$
\begin{aligned}
\hat{\mathbb{X}}|x\rangle & =x|x\rangle, \\
\hat{\mathbb{P}}_{x}\left|p_{x}\right\rangle & =p_{x}\left|p_{x}\right\rangle .
\end{aligned}
$$

Donde $x\left(p_{x}\right)$ es el valor propio de posición (momento) asociado al estado propio $|x\rangle\left(\left|p_{x}\right\rangle\right)$.

La experiencia muestra que la posición (momento) de la partícula puede ser cualquier valor real, por tanto los valores propios de $\hat{\mathbb{X}}\left(\hat{\mathbb{P}}_{x}\right)$ forman un conjunto continuo. Así, el conjunto de estados propios $\{|x\rangle\}\left(\left\{\left|p_{x}\right\rangle\right\}\right)$ constituye una base continua ortonormal y completa.

$$
\begin{gathered}
\left\langle x \mid x^{\prime}\right\rangle=\delta\left(x-x^{\prime}\right), \quad\left\langle p_{x} \mid p_{x}^{\prime}\right\rangle=\delta\left(p_{x}-p_{x}^{\prime}\right), \\
\int_{-\infty}^{\infty} d x|x\rangle\left\langle x\left|=\hat{\mathbb{I}}_{x}, \quad \int_{-\infty}^{\infty} d p_{x}\right| p_{x}\right\rangle\left\langle p_{x}\right|=\hat{\mathbb{I}}_{p_{x}},
\end{gathered}
$$

donde $\delta\left(x-x^{\prime}\right)$ y $\delta\left(p_{x}-p_{x}^{\prime}\right)$ son deltas de Dirac. $\hat{\mathbb{I}}_{x}$ y $\hat{\mathbb{I}}_{p_{x}}$ son operadores identidad en cada base. Además,

$$
\left\langle x \mid p_{x}\right\rangle=\frac{1}{\sqrt{2 \pi \hbar}} e^{\frac{i}{\hbar} p_{x} x}
$$

\subsection{Funciones de Onda}

Un estado arbitrario $|\psi\rangle$ puede ser escrito como combinación lineal de los estados de la base de posición o de momento.

En la base de posición

$$
|\psi\rangle=\hat{\mathbb{I}}_{x}|\psi\rangle=\int_{-\infty}^{\infty} d x \psi(x)|x\rangle,
$$

donde a

$$
\psi(x)=\langle x \mid \psi\rangle
$$

se le llama función de onda, en la representación de posición, de la partícula que se encuentra en el estado $|\psi\rangle$.

Similarmente, en la base de momento

$$
|\psi\rangle=\hat{\mathbb{I}}_{p_{x}}|\psi\rangle=\int_{-\infty}^{\infty} d p_{x} \phi\left(p_{x}\right)\left|p_{x}\right\rangle,
$$

donde a

$$
\phi\left(p_{x}\right)=\left\langle p_{x} \mid \psi\right\rangle
$$

se le llama función de onda, en la representación de momento lineal.

\subsection{Relación entre las Funciones $\psi(x)$ y $\phi\left(p_{x}\right)$}

Usando las ecuaciones (12), (7b) y (8)

$$
\phi\left(p_{x}\right)=\left\langle p_{x}\left|\hat{\mathbb{I}}_{x}\right| \psi\right\rangle=\frac{1}{\sqrt{2 \pi \hbar}} \int_{-\infty}^{\infty} e^{-\frac{i}{\hbar} p_{x} x} \psi(x) d x .
$$

Similarmente, usando (10), (7b) y (8)

$$
\psi(x)=\left\langle x\left|\hat{\mathbb{I}}_{p_{x}}\right| \psi\right\rangle=\frac{1}{\sqrt{2 \pi \hbar}} \int_{-\infty}^{\infty} e^{\frac{i}{\hbar} p_{x} x} \phi\left(p_{x}\right) d p_{x} .
$$

En las cuales se nota que $\psi(x)$ y $\phi\left(p_{x}\right)$ están relacionadas a través de la transformada de Fourier.

\subsection{Funciones de Operadores}

Si $\hat{\mathbb{F}}(\hat{\mathbb{X}})$ y $\hat{\mathbb{G}}\left(\hat{\mathbb{P}}_{x}\right)$ son funciones de $\hat{\mathbb{X}}$ y $\hat{\mathbb{P}}_{x}$, entonces en la base de posición

$$
\begin{aligned}
& \langle x|\hat{\mathbb{X}}| \psi\rangle=x \psi(x), \\
& \langle x|\hat{\mathbb{F}}(\hat{\mathbb{X}})| \psi\rangle=f(x) \psi(x), \\
& \left\langle x\left|\hat{\mathbb{P}}_{x}\right| \psi\right\rangle=-i \hbar \frac{d}{d x} \psi(x), \\
& \left\langle x\left|\hat{\mathbb{G}}\left(\hat{\mathbb{P}}_{x}\right)\right| \psi\right\rangle=\frac{1}{\sqrt{2 \pi \hbar}} \int_{-\infty}^{\infty} d x^{\prime} \tilde{g}\left(x-x^{\prime}\right) \psi\left(x^{\prime}\right) .
\end{aligned}
$$

Donde $f(x)$ es una función de variable real asociada a $\hat{\mathbb{F}}(\hat{\mathbb{X}})$,

$$
f(x)=\langle x| \hat{\mathbb{F}}(\hat{\mathbb{X}}),
$$

$\tilde{g}(x)$ es la transformada inversa de Fourier de la función de variable real $g\left(p_{x}\right)$, asociada a $\hat{\mathbb{G}}_{\left(\hat{\mathbb{P}}_{x}\right)}$,

$$
\tilde{g}(x)=\frac{1}{\sqrt{2 \pi \hbar}} \int_{-\infty}^{\infty} d p_{x} g\left(p_{x}\right) e^{\frac{i}{\hbar} p_{x} x} .
$$

De (15a) y (15b) se nota que, en la base de posición, el operador $\hat{\mathbb{X}}$ se comporta como una variable real y $\hat{\mathbb{F}}(\hat{\mathbb{X}})$ como una función de esta variable

$$
\begin{gathered}
\hat{\mathbb{X}} \longrightarrow x, \\
\hat{\mathbb{F}}(\hat{\mathbb{X}}) \longrightarrow f(x) .
\end{gathered}
$$

Además de (15c) se concluye que el operador $\hat{\mathbb{P}}_{x}$ actúa como un operador diferencial

$$
\hat{\mathbb{P}}_{x} \longrightarrow-i \hbar \frac{d}{d x} .
$$


En la base de momento

$$
\begin{aligned}
\left\langle p_{x}|\hat{\mathbb{X}}| \psi\right\rangle & =i \hbar \frac{d}{d p_{x}} \phi\left(p_{x}\right), \\
\left\langle p_{x}|\hat{\mathbb{F}}(\hat{\mathbb{X}})| \psi\right\rangle & =\frac{1}{\sqrt{2 \pi \hbar}} \int_{-\infty}^{\infty} d p_{x}^{\prime} \bar{f}\left(p_{x}-p_{x}^{\prime}\right) \phi\left(p_{x}^{\prime}\right), \\
\left\langle p_{x}\left|\hat{\mathbb{P}}_{x}\right| \psi\right\rangle & =p_{x} \phi\left(p_{x}\right), \\
\left\langle p_{x}\left|\hat{\mathbb{G}}_{(}\left(\hat{\mathbb{P}}_{x}\right)\right| \psi\right\rangle & =g\left(p_{x}\right) \phi\left(p_{x}\right),
\end{aligned}
$$

donde $\bar{f}\left(p_{x}\right)$ es la tranformada de Fourier de $f(x)$

$$
\bar{f}\left(p_{x}\right)=\frac{1}{\sqrt{2 \pi \hbar}} \int_{-\infty}^{\infty} d x f(x) e^{-\frac{i}{\hbar} x p_{x}},
$$

y $g\left(p_{x}\right)$ está dada por

$$
g\left(p_{x}\right)=\left\langle p_{x}\right| \hat{\mathbb{G}}\left(\hat{\mathbb{P}}_{x}\right)
$$

Ahora, a partir de (20a) se nota que, en la base de momento, el operador $\hat{\mathbb{X}}$ actúa como un operador diferencial

$$
\hat{\mathbb{X}} \longrightarrow i \hbar \frac{d}{d p_{x}} .
$$

El operador $\hat{\mathbb{P}}_{x}$ se comporta como una variable real y $\hat{\mathbb{G}}\left(\hat{\mathbb{P}}_{x}\right)$ como una función de dicha variable

$$
\begin{gathered}
\hat{\mathbb{P}}_{x} \longrightarrow p_{x}, \\
\hat{\mathbb{G}}\left(\hat{\mathbb{P}}_{x}\right) \longrightarrow g\left(p_{x}\right) .
\end{gathered}
$$

\subsection{Ecuacion de Schrödinger Estacionaria}

En la representación de posición, la ecuación (4), en el caso unidimensional, queda escrita como

$$
\begin{aligned}
& \left\langle x|\hat{\mathbb{H}}| \psi_{n}\right\rangle=\left\langle x\left|\left(\frac{\hat{\mathbb{P}}_{x}^{2}}{2 m}+\hat{\mathbb{V}}(\hat{\mathbb{X}})\right)\right| \psi_{n}\right\rangle=E_{n}\left\langle x \mid \psi_{n}\right\rangle \\
& -\frac{\hbar^{2}}{2 m} \frac{d^{2} \psi_{n}(x)}{d x^{2}}+V(x) \psi_{n}(x)=E_{n} \psi_{n}(x),
\end{aligned}
$$

donde se ha usado (15a)-(15c).

En la representación de momento

$$
\begin{aligned}
& \left\langle p_{x}|\hat{\mathbb{H}}| \psi_{n}\right\rangle=\left\langle p_{x}\left|\left(\frac{\hat{\mathbb{P}}_{x}^{2}}{2 m}+\hat{\mathbb{V}}(\hat{\mathbb{X}})\right)\right| \psi_{n}\right\rangle=E_{n}\left\langle p_{x} \mid \psi_{n}\right\rangle, \\
& \begin{aligned}
\frac{p_{x}^{2}}{2 m} \phi_{n}\left(p_{x}\right)+\frac{1}{\sqrt{2 \pi \hbar}} \int_{-\infty}^{\infty} d p_{x}^{\prime} \bar{V}\left(p_{x}-p_{x}^{\prime}\right) \phi_{n}\left(p_{x}^{\prime}\right) \\
\left.=E_{n} \phi_{n}\left(p_{x}\right), \quad \text { (26a) }\right)
\end{aligned}
\end{aligned}
$$

donde hemos usado (20a)-(20c) y según (21),

$$
\bar{V}\left(p_{x}-p_{x}^{\prime}\right)=\frac{1}{\sqrt{2 \pi \hbar}} \int_{-\infty}^{\infty} d x V(x) e^{-\frac{i}{\hbar} x\left(p_{x}-p_{x}^{\prime}\right)} .
$$

\section{Ejemplos}

En esta sección, resolvemos la ecuación de Schrödinger en las representaciones de posición y de momento, es decir, encontramos las funciones de onda y los valores propios de energía en los siguientes casos.

1. Partícula en un potencial lineal

2. Oscilador armónico

3. Partícula en un potencial delta de Dirac

Además, verificamos las ecuacionnes (13) y (14) que permiten encontrar $\phi(p)$ a partir $\psi(x)$ o visceversa.

\subsection{Partícula en un Potencial Lineal}

Consideremos una partícula de masa $m$ sometida a un potencial

$$
\hat{\mathbb{V}}(\hat{\mathbb{X}})=a \hat{\mathbb{X}}
$$

donde $a$ es una constante positiva.

\section{Representación de Posición}

En este caso, según (16)

$$
V(x)=\langle x| \hat{\mathbb{V}}(\hat{\mathbb{X}})=a x,
$$

con lo que la ecuación de Schrödinger (25a) se puede escribir como

$$
-\frac{\hbar^{2}}{2 m} \frac{d^{2} \psi_{n}(x)}{d x^{2}}+a x \psi_{n}(x)=E_{n} \psi_{n}(x)
$$

Reorganizando términos

$$
\frac{d^{2} \psi_{n}(x)}{d x^{2}}+\frac{2 m}{\hbar^{2}}(E-a x) \psi_{n}(x)=0
$$

cuya solución queda escrita en términos de las funciones de Ayri

$$
\begin{aligned}
\psi_{n}(x)=D & \operatorname{Ai}\left[\left(\frac{2 m a}{\hbar^{2}}\right)^{1 / 3}\left(x-\frac{E}{a}\right)\right] \\
+F & \operatorname{Bi}\left[\left(\frac{2 m a}{\hbar^{2}}\right)^{1 / 3}\left(x-\frac{E}{a}\right)\right] .
\end{aligned}
$$

Como $\operatorname{Ai}(x \rightarrow \infty) \rightarrow 0$ y $\operatorname{Bi}(x \rightarrow \infty) \rightarrow \infty$, la condición de frontera $\psi_{n}(x \rightarrow \infty) \rightarrow 0$ requiere que $F=0$. Por tanto

$$
\psi_{n}(x)=D \text { Ai }\left[\left(\frac{2 m a}{\hbar^{2}}\right)^{1 / 3}\left(x-\frac{E}{a}\right)\right] .
$$


Usando la representación integral de la función de Airy [10], expresando la exponencial compleja en términos de funciones seno y coseno, usando las propiedades de paridad

$$
\begin{aligned}
& \int_{-\infty}^{\infty} d u \exp \left[i\left(\beta u+\frac{u^{3}}{3}\right)\right] \\
& \quad=2 \int_{0}^{\infty} d u \cos \left(\beta u+\frac{u^{3}}{3}\right)=2 \pi \mathrm{Ai}(\beta) .
\end{aligned}
$$

$\operatorname{con} \beta=\left(\frac{2 m a}{\hbar^{2}}\right)^{1 / 3}\left(x-\frac{E}{a}\right)$ podemos escribir

$$
\begin{aligned}
\psi_{n}(x)=\frac{D}{2 \pi} & \int_{-\infty}^{\infty} d u \exp \left[i \left(\frac{u^{3}}{3}\right.\right. \\
& \left.\left.+\left(\frac{2 m a}{\hbar^{2}}\right)^{1 / 3}\left(x-\frac{E}{a}\right) u\right)\right] .
\end{aligned}
$$

La constante $D$ se halla con la condición de normalización [11]

$$
\begin{gathered}
\int_{-\infty}^{\infty} d x \psi_{n}^{*}(x) \psi_{n^{\prime}}(x)=\delta\left(E_{n}-E_{n^{\prime}}\right) \\
\frac{|D|^{2} a}{4 \pi^{2}}\left(\frac{\hbar^{2}}{2 m a}\right)^{2 / 3} \delta\left(E_{n}-E_{n^{\prime}}\right)=\delta\left(E_{n}-E_{n^{\prime}}\right)
\end{gathered}
$$

escogiendo $D$ real y positiva

$$
D=\frac{2 \pi}{\sqrt{a}}\left(\frac{2 m a}{\hbar^{2}}\right)^{1 / 3}
$$

donde hemos usado

$$
\begin{gathered}
\int_{-\infty}^{\infty} d x \psi_{n}^{*}(x) \psi_{n^{\prime}}(x)=\frac{|D|^{2}}{4 \pi^{2}} \int_{-\infty}^{\infty} d x \\
\times \int_{-\infty}^{\infty} d u \exp \left[-i\left(\frac{u^{3}}{3}+\left(\frac{2 m a}{\hbar^{2}}\right)^{1 / 3}\left(x-\frac{E_{n}}{a}\right) u\right)\right] \\
\times \int_{-\infty}^{\infty} d u^{\prime} \exp \left[i\left(\frac{u^{\prime 3}}{3}+\left(\frac{2 m a}{\hbar^{2}}\right)^{1 / 3}\left(x-\frac{E_{n^{\prime}}}{a}\right) u^{\prime}\right)\right] \\
=\frac{|D|^{2} a}{4 \pi^{2}}\left(\frac{\hbar^{2}}{2 m a}\right)^{2 / 3} \delta\left(E_{n}-E_{n^{\prime}}\right) .
\end{gathered}
$$

Por tanto (35) queda

$$
\begin{aligned}
\psi_{n}(x)=\frac{1}{\sqrt{a}} & \left(\frac{2 m a}{\hbar^{2}}\right)^{1 / 3} \\
& \times \operatorname{Ai}\left[\left(\frac{2 m a}{\hbar^{2}}\right)^{1 / 3}\left(x-\frac{E}{a}\right)\right] .
\end{aligned}
$$

\section{Representación de Momento}

Teniendo en cuenta (27), o reemplazando directamente (23), la ecuación de Schrödinger (26a) se puede escribir como

$$
\frac{p_{x}^{2}}{2 m} \phi_{n}\left(p_{x}\right)+i a \hbar \frac{d \phi_{n}\left(p_{x}\right)}{d p_{x}}=E_{n} \phi_{n}\left(p_{x}\right) .
$$

Reorganizando términos

$$
\frac{d \phi_{n}\left(p_{x}\right)}{d p_{x}}-\frac{i}{a \hbar}\left(\frac{p_{x}^{2}}{2 m}-E_{n}\right) \phi_{n}\left(p_{x}\right)=0
$$

La solución es de la forma

$$
\phi_{n}\left(p_{x}\right)=A \exp \left[\frac{i}{a \hbar}\left(\frac{p_{x}^{3}}{6 m}-E_{n} p_{x}\right)\right],
$$

donde $A$ es la constante de integración, que podemos encontrar a partir de la condición de nomalización de la función de onda [11]

$$
\begin{aligned}
\int_{-\infty}^{\infty} d p_{x} \phi_{n}^{*}\left(p_{x}\right) \phi_{n^{\prime}}\left(p_{x}\right) & =\delta\left(E_{n}-E_{n^{\prime}}\right) \\
|A|^{2} \int_{-\infty}^{\infty} d p_{x} \exp \left[\frac{i p_{x}}{a \hbar}\left(E_{n}-E_{n^{\prime}}\right)\right] & =\delta\left(E_{n}-E_{n^{\prime}}\right) \\
2 \pi \hbar a \delta\left(E_{n}-E_{n^{\prime}}\right)|A|^{2} & =\delta\left(E_{n}-E_{n^{\prime}}\right) \\
|A|^{2} & =\frac{1}{2 \pi \hbar a},
\end{aligned}
$$

escogiendo $A$ real y positivo

$$
A=\frac{1}{\sqrt{2 \pi \hbar a}} .
$$

Así, la función de onda (43) queda

$$
\phi_{n}\left(p_{x}\right)=\frac{1}{\sqrt{2 \pi \hbar a}} \exp \left[\frac{i}{a \hbar}\left(\frac{p_{x}^{3}}{6 m}-E_{n} p_{x}\right)\right] .
$$

\section{Transformada de Fourier}

Podemos encontrar la función de onda $\psi_{n}(x)$ a partir de $\phi_{n}\left(p_{x}\right)$ usando la transformada de Fourier (14)

$$
\psi_{n}(x)=\frac{1}{\sqrt{2 \pi \hbar}} \int_{-\infty}^{\infty} d p_{x} e^{\frac{i}{\hbar} p_{x} x} \phi_{n}\left(p_{x}\right)
$$

$$
\begin{aligned}
\psi_{n}(x)=\frac{1}{2 \pi \hbar \sqrt{a}} \int_{-\infty}^{\infty} d p_{x} \exp \left[i \frac{p_{x}^{3}}{6 a \hbar m}\right. \\
\left.+\frac{i}{\hbar} p_{x}\left(x-\frac{E_{n}}{a}\right)\right]
\end{aligned}
$$


Haciendo el cambio de variable

$$
u=\left(\frac{1}{2 m a \hbar}\right)^{1 / 3} p_{x}
$$

y definiendo

$$
\alpha=\left(\frac{2 m a}{\hbar^{2}}\right)^{1 / 3}, \quad \beta=\left(x-\frac{E}{a}\right) \alpha
$$

la ecuación (67) se puede escribir como

$$
\psi_{n}(x)=\frac{\alpha}{2 \pi \sqrt{a}} \int_{-\infty}^{\infty} d u \exp \left[i\left(\beta u+\frac{u^{3}}{3}\right)\right]
$$

Expresando la exponencial compleja en términos de funciones seno y coseno, usando las propiedades de paridad y la representación integral de la función de Airy [10],

$$
\begin{aligned}
& \int_{-\infty}^{\infty} d u \exp \left[i\left(\beta u+\frac{u^{3}}{3}\right)\right] \\
& \quad=2 \int_{0}^{\infty} d u \cos \left(\beta u+\frac{u^{3}}{3}\right)=2 \pi \mathrm{Ai}(\beta) .
\end{aligned}
$$

Por tanto la ecuación (50) queda

$$
\psi_{n}(x)=\frac{\alpha}{\sqrt{a}} \operatorname{Ai}(\beta)
$$

Para finalizar, usando las definiciones de $\alpha$ y $\beta$ se tiene

$$
\begin{aligned}
\psi_{n}(x)=\frac{1}{\sqrt{a}} & \left(\frac{2 m a}{\hbar^{2}}\right)^{1 / 3} \\
& \times \mathrm{Ai}\left[\left(\frac{2 m a}{\hbar^{2}}\right)^{1 / 3}\left(x-\frac{E}{a}\right)\right]
\end{aligned}
$$

la cual coincide, como debe ser, con la ecuación (40) hallada al solucionar la ecuación de Schrödinger en la representación de coordenadas.

\subsection{Oscilador Armónico}

Consideremos una partícula de masa $m$ y frecuencia angular $\omega$ sometida a un potencial

$$
\hat{\mathbb{V}}(\hat{\mathbb{X}})=\frac{1}{2} k \hat{\mathbb{X}}^{2}
$$

donde $k=m \omega^{2}$ es una constante positiva.

\section{Representación de Posición}

En este caso, según (16)

$$
V(x)=\langle x| \hat{\mathbb{V}}(\hat{\mathbb{X}})=\frac{1}{2} k x^{2}=\frac{1}{2} m \omega^{2} x^{2},
$$

con lo que la ecuación de Schrödinger (25a) se puede escribir como

$$
-\frac{\hbar^{2}}{2 m} \frac{d^{2} \psi_{n}(x)}{d x^{2}}+\frac{1}{2} m \omega^{2} x^{2} \psi_{n}(x)=E_{n} \psi_{n}(x)
$$

Reorganizando términos

$$
\frac{d^{2} \psi_{n}(x)}{d x^{2}}-\frac{m^{2} \omega^{2}}{\hbar^{2}} x^{2} \psi_{n}(x)=-\frac{2 m E}{\hbar^{2}} \psi_{n}(x) .
$$

Siguiendo el procedimiento tradicional de los textos de Mecánica Cuántica [12, 13, 14] es conveniente adimensionar esta ecuación proponiendo $x=\alpha u$, donde $u$ es adimensional y $\alpha$, que tiene unidades de distancia, está dado por

$$
\alpha=\sqrt{\frac{\hbar}{m \omega}} .
$$

De mamera que al reemplazar en (57) se tiene

$$
\frac{d^{2} \psi_{n}(u)}{d u^{2}}+\left(\varepsilon-u^{2}\right) \psi_{n}(u)=0
$$

donde $\varepsilon=\frac{2 E}{\hbar \omega}$ es una cantidad adimensional.

La solución de (59) es

$$
\psi_{n}(u)=B_{n} \mathrm{H}_{n}(u) e^{-\frac{1}{2} u^{2}},
$$

donde $\mathrm{H}_{n}(u)$ es un polinomio de Hermite de grado $n(=0,1,2, \ldots), B_{n}$ se halla normalizando y además, se tiene la restricción $\varepsilon-1=2 n$.

Escribiendo (60) en términos de $x$ y normalizando

$$
\begin{aligned}
\psi_{n}(x)= & \frac{1}{\sqrt{2^{n} n !}}\left(\frac{m \omega}{\pi \hbar}\right)^{1 / 4} \\
& \times \exp \left(-\frac{m \omega}{2 \hbar} x^{2}\right) \mathrm{H}_{n}\left(\sqrt{\frac{m \omega}{\hbar}} x\right)
\end{aligned}
$$

\section{Representación de Momento}

Teniendo en cuenta (27), o reemplazando directamente (23), la ecuación de Schrödinger (26a) se puede escribir como

$$
\frac{p_{x}^{2}}{2 m} \phi_{n}\left(p_{x}\right)-\frac{1}{2} m \omega^{2} \hbar^{2} \frac{d^{2} \phi_{n}\left(p_{x}\right)}{d p_{x}^{2}}=E_{n} \phi_{n}\left(p_{x}\right) .
$$


Reorganizando términos

$$
\begin{aligned}
\frac{d^{2} \phi_{n}\left(p_{x}\right)}{d p_{x}^{2}}-\frac{1}{m^{2} \omega^{2} \hbar^{2}} p_{x}^{2} \phi_{n}\left(p_{x}\right) & \\
& =-\frac{2 E_{n}}{m \omega^{2} \hbar^{2}} \phi_{n}\left(p_{x}\right) .
\end{aligned}
$$

Para adimensionar, se hace el cambio de variable $p_{x}=\beta v=\sqrt{m \omega \hbar} v$ y $\varepsilon=\frac{2 E_{n}}{\hbar \omega}$ con lo cual la ecuación (63) queda

$$
\frac{d^{2} \phi_{n}(v)}{d v^{2}}-v^{2} \phi_{n}(v)=-\varepsilon \phi_{n}(v) .
$$

La ecuación diferencial (64) tiene la misma estructura matemática de la ecuación (59), por tanto su solución será

$$
\phi_{n}(v)=C_{n} \mathrm{H}_{n}(v) e^{-\frac{1}{2} v^{2}} .
$$

Regresando a la variable $p_{x}$ y normalizando

$$
\begin{aligned}
& \phi_{n}\left(p_{x}\right)=\frac{1}{\sqrt{2^{n} n !}}\left(\frac{1}{\pi m \omega \hbar}\right)^{1 / 4} \\
& \times \exp \left(-\frac{1}{2 m \omega \hbar} p_{x}^{2}\right) \mathrm{H}_{n}\left(\frac{1}{\sqrt{m \omega \hbar}} p_{x}\right)
\end{aligned}
$$

\section{Transformada de Fourier}

Podemos encontrar la función de onda $\psi_{n}(x)$ a partir de $\phi_{n}\left(p_{x}\right)$ usando la transformada de Fourier (14)

$$
\begin{array}{r}
\psi_{n}(x)=\frac{1}{\sqrt{2 \pi \hbar}} \int_{-\infty}^{\infty} d p_{x} e^{\frac{i}{\hbar} p_{x} x} \phi_{n}\left(p_{x}\right) \\
\psi_{n}(x)=\frac{1}{\sqrt{2 \pi \hbar}} \frac{1}{\sqrt{2^{n} n !}}\left(\frac{1}{\pi m \omega \hbar}\right)^{1 / 4} \\
\times \int_{-\infty}^{\infty} d p_{x} \exp \left(\frac{i}{\hbar} p_{x} x\right) \\
\times \exp \left(-\frac{1}{2 m \omega \hbar} p_{x}^{2}\right) \mathrm{H}_{n}\left(\frac{1}{\sqrt{m \omega \hbar}} p_{x}\right) .
\end{array}
$$

Para abreviar definamos $\gamma=\frac{1}{\sqrt{m \omega \hbar}} \mathrm{y} \tau=\frac{x}{\alpha \hbar}$ con lo cual

$$
\begin{aligned}
\psi_{n}(x)=\frac{1}{\sqrt{2 \pi \hbar}} \frac{1}{\sqrt{2^{n} n !}}\left(\frac{1}{\pi m \omega \hbar}\right)^{1 / 4} \\
\quad \int_{-\infty}^{\infty} d p_{x} e^{i \tau \gamma p_{x}} e^{-\frac{1}{2} \gamma^{2} p_{x}^{2}} \mathrm{H}_{n}\left(\gamma p_{x}\right) .
\end{aligned}
$$

Ahora, haciendo el cambio de variable $\mu=\gamma p_{x}$

$$
\begin{aligned}
\psi_{n}(x)=\frac{1}{\sqrt{2 \pi \hbar}} & \frac{1}{\sqrt{2^{n} n !}}\left(\frac{1}{\pi m \omega \hbar}\right)^{1 / 4} \frac{1}{\gamma} \\
& \int_{-\infty}^{\infty} d p_{x} e^{i \tau \mu} e^{-\frac{1}{2} \mu^{2}} \mathrm{H}_{n}(\mu) .
\end{aligned}
$$

Según Gradshteyn and Ryzhik,[15]

$$
\begin{aligned}
\int_{-\infty}^{\infty} d p_{x} e^{i \tau \mu} e^{-\frac{1}{2} \mu^{2}} \mathrm{H}_{n}(\mu) & \\
& =\sqrt{2 \pi} e^{-\frac{1}{2} \tau^{2}} \mathrm{H}_{n}(\tau) i^{n},
\end{aligned}
$$

por tanto

$$
\begin{aligned}
\psi_{n}(x)=\frac{1}{\sqrt{2 \pi \hbar}} \frac{1}{\sqrt{2^{n} n !}}\left(\frac{1}{\pi m \omega \hbar}\right)^{1 / 4} \\
\frac{1}{\gamma} \sqrt{2 \pi} e^{-\frac{1}{2} \tau^{2}} \mathrm{H}_{n}(\tau) i^{n} .
\end{aligned}
$$

Finalmente, reemplazando $\gamma$ y $\tau$

$$
\begin{array}{r}
\psi_{n}(x)=\frac{1}{\sqrt{2^{n} n !}}\left(\frac{m \omega}{\pi \hbar}\right)^{1 / 4} \exp \left(-\frac{m \omega}{2 \hbar} x^{2}\right) \\
\mathrm{H}_{n}\left(\sqrt{\frac{m \omega}{\hbar}} x\right) i^{n} .
\end{array}
$$

donde $i^{n}$ es un factor de fase que no afecta la norma de la función de onda, por tanto podemos concluir que las funciones de onda (72) y (61) representan el mismo estado.

\subsection{Potencial Delta de Dirac}

Ilustramos el caso de un potencial tipo Delta de Dirac considerando los estados ligados de una partícula de masas $m$ sometida a un potencial atractivo de la forma $[12,3]$

$$
\hat{\mathbb{V}}(\hat{\mathbb{X}})=-V_{0} \delta(\hat{\mathbb{X}}) \hat{\mathbb{I}}
$$

donde $V_{0}$ es una constante positiva.

\section{Representación de Posición}

Según (16), el potencial se puede escribir como

$$
V(x)=\langle x| \hat{\mathbb{V}}(\hat{\mathbb{X}})=-V_{0} \delta(x),
$$

con lo cual la ecuación de Schrödinger estacionaria (25a) toma la forma

$$
-\frac{\hbar^{2}}{2 m} \frac{d^{2} \psi_{n}(x)}{d x^{2}}-V_{0} \delta(x) \psi_{n}(x)=E_{n} \psi_{n}(x) .
$$


Reorganizando términos

$$
\frac{d^{2} \psi_{n}(x)}{d x^{2}}+\frac{2 m V_{0}}{\hbar^{2}} \delta(x) \psi_{n}(x)=-\frac{2 m E}{\hbar^{2}} \psi_{n}(x) .
$$

Como estamos considerando estados ligados, escribi$\operatorname{mos} E=-|E|$ y definimos $k^{2}=\frac{2 m|E|}{\hbar^{2}}$, con lo cual la ecuación (76) se transforma en

$$
\frac{d^{2} \psi_{n}(x)}{d x^{2}}+\frac{2 m V_{0}}{\hbar^{2}} \delta(x) \psi_{n}(x)=k^{2} \psi_{n}(x) .
$$

Para $x<0, \delta(x)=0$ y la solución (77) es

$$
\psi_{n 1}(x)=A e^{-k x}+B e^{k x} .
$$

Pero la condición de frontera cuando $x \rightarrow-\infty$ obliga a que $A=0$, por tanto

$$
\psi_{n 1}(x)=B e^{k x}, \quad(x<0) .
$$

Similarmente, para $x>0, \delta(x)=0$ y (77) tiene como solución

$$
\psi_{n 2}(x)=C e^{-k x}+D e^{k x} .
$$

La condición de frontera cuando $x \rightarrow \infty$ exige que $D=0$, por tanto

$$
\psi_{n 2}(x)=C e^{-k x}, \quad(x>0) .
$$

Para encontrar la energía y los valores de $B$ y $C$, primero usamos la condición de continuidad de la función de onda en $x=0$

$$
\psi_{n 1}(0)=\psi_{n 2}(0)
$$

que conduce a

$$
B=C \text {. }
$$

Así, podemos agrupar (79) y (81) como

$$
\psi_{n}(x)=C e^{-k|x|} .
$$

Como segundo paso consideramos la primera derivada de la función de onda. Aunque $d \psi_{n}(x) / d x$ no es continua en $x=0$, la ecuación de Schrödinger (77) se puede integrar en un intervalo muy pequeño $[-\varepsilon,+\varepsilon]$ alrededor de $x=0$

$$
\begin{array}{r}
\int_{-\varepsilon}^{+\varepsilon} \frac{d^{2} \psi_{n}(x)}{d x^{2}} d x+\frac{2 m V_{0}}{\hbar^{2}} \int_{-\varepsilon}^{+\varepsilon} \delta(x) \psi_{n}(x) d x \\
=k^{2} \int_{-\varepsilon}^{+\varepsilon} \psi_{n}(x) d x .
\end{array}
$$

Si usamos el hecho que en el intervalo $[-\varepsilon,+\varepsilon]$ $\psi_{n}(x) \approx \psi_{n}(0)$ se obtiene

$$
k=\frac{m V_{0}}{\hbar^{2}}
$$

y la energía será

$$
E=-|E|=-\frac{\hbar^{2} k^{2}}{2 m}=-\frac{m V_{0}^{2}}{2 \hbar^{2}} .
$$

Entonces, reemplazando en (84)

$$
\psi_{n}(x)=C \exp \left(-\frac{m V_{0}}{\hbar^{2}}|x|\right) .
$$

Finalmente normalizamos y encontramos que

$$
C=\frac{\sqrt{m V_{0}}}{\hbar}
$$

y por tanto,

$$
\psi_{n}(x)=\frac{\sqrt{m V_{0}}}{\hbar} \exp \left(-\frac{m V_{0}}{\hbar^{2}}|x|\right) .
$$

\section{Representación de Momento}

La ecuación de Schrödinger (26a) se puede escribir como

$$
\begin{aligned}
& \left(p_{x}^{2}-2 m E\right) \phi_{n}\left(p_{x}\right) \\
& +\sqrt{\frac{2}{\pi \hbar}} m \int_{-\infty}^{\infty} d p_{x}^{\prime} \bar{V}\left(p_{x}-p_{x}^{\prime}\right) \phi_{n}\left(p_{x}^{\prime}\right)=0 .
\end{aligned}
$$

Donde, usando (27)

$$
\bar{V}\left(p_{x}-p_{x}^{\prime}\right)=-\frac{V_{0}}{\sqrt{2 \pi \hbar}} .
$$

Además, considerando estados ligados $(E=-|E|)$ obtenemos

$$
\left(p_{x}^{2}+2 m|E|\right) \phi_{n}\left(p_{x}\right)-\frac{m V_{0}}{\pi \hbar} \int_{-\infty}^{\infty} d p_{x}^{\prime} \phi_{n}\left(p_{x}^{\prime}\right)=0 .
$$

Para solucionar (93) se hace el cambio

$$
\alpha=\frac{m V_{0}}{\pi \hbar} \int_{-\infty}^{\infty} d p_{x}^{\prime} \phi_{n}\left(p_{x}^{\prime}\right)
$$

Así que, a partir de (93)

$$
\phi_{n}\left(p_{x}\right)=\frac{\alpha}{p_{x}^{2}+2 m|E|} .
$$


Ahora, al reemplazar (95) en (94) y realizando la integración

$$
\alpha=\frac{m V_{0} \alpha}{\hbar \sqrt{2 m|E|}}
$$

con lo cual

$$
|E|=\frac{m V_{0}^{2}}{2 \hbar^{2}}
$$

y la energía estará dada, como ya se habia obtenido en (87), por

$$
E=-|E|=-\frac{m V_{0}^{2}}{2 \hbar^{2}}
$$

Reemplazando (97) en (95)

$$
\phi_{n}\left(p_{x}\right)=\frac{\alpha}{p_{x}^{2}+\left(\frac{m V_{0}}{\hbar}\right)^{2}} .
$$

Al normalizar se encuentra

$$
\alpha=\sqrt{\frac{2}{\pi}}\left(\frac{m V_{0}}{\hbar}\right)^{3 / 2},
$$

donde usamos la integral 17.6.8 de Spiegel [16]. Por tanto, a partir de (99), la función de onda en la representación de momento queda de la forma

$$
\phi_{n}\left(p_{x}\right)=\sqrt{\frac{2}{\pi}}\left(\frac{m V_{0}}{\hbar}\right)^{3 / 2} \frac{1}{p_{x}^{2}+\left(\frac{m V_{0}}{\hbar}\right)^{2}} .
$$

\section{Transformada de Fourier}

Partiendo de la función de onda (101) obtenemos $\psi_{n}(x)$ usando la transformada de Fourier (14). Notemos que

$$
\psi_{n}(x)=\frac{\left(m V_{0}\right)^{3 / 2}}{\pi \hbar^{2}} \int_{-\infty}^{\infty} d p_{x} \frac{e^{\frac{i}{\hbar} p_{x} x}}{p_{x}^{2}+\left(\frac{m V_{0}}{\hbar}\right)^{2}}
$$

Al utilizar el software Mathematica 8 encontramos que la integral es

$$
\int_{-\infty}^{\infty} d p_{x} \frac{e^{\frac{i}{\hbar} p_{x} x}}{p_{x}^{2}+\left(\frac{m V_{0}}{\hbar}\right)^{2}}=\frac{\pi \hbar}{m V_{0}} e^{-\frac{m V_{0}}{\hbar^{2}}|x|}
$$

por lo tanto obtenemos, como en (90), que la función de onda en representación de posición es

$$
\psi_{n}(x)=\frac{\sqrt{m V_{0}}}{\hbar} \exp \left(-\frac{m V_{0}}{\hbar^{2}}|x|\right)
$$

como en la ecuación (90).

\section{Conclusiones}

Hemos ilustrado el uso y conexión de las representaciones de coordenadas y de momentos en mecánica cuántica a través de tres ejemplos elementales. Esperamos que este trabajo permita comprender que ambas representaciones poseen el mismo estatus teórico y son dos aspectos complementarios con los que podemos describir el estado de un sistema cuántico.

\section{Referencias}

[1] J. J. Sakurai. Modern Quantum Mechanics. Addison-Wesley Pub. Co, rev. edition, 1994.

[2] C. Cohen-Tannoudji, B. Diu, and F. Laloe. Quantum Mechanics, volume 1. John Wiley and Sons, 1 edition, 1977.

[3] D. McMahon. Quantum Mechanics DeMYSTiFieD. McGraw-Hill, 3 edition, 2006.

[4] Juan Carlos López Vieyra. Introducción a la mecánica cuántica, Jul. 1997. Instituto de Ciencias Nucleares de la UNAM, https://www.nucleares.unam.mx/ vieyra/cuant1.html.

[5] Richard Fitzpatrick. Quantum mechanics, Jul. 2010. The University of Texas at Austin, http://farside.ph.utexas.edu/teaching/qmech/Quantum/Quantum.html.

[6] E. Guillaumín-España, A. L. Salas-Brito, R. P. Martínez y Romero, and H. N. NúñezYépez. Simple quantum systems in the momentum representation. Rev. Mex. Fis., 47(1) p. $98,2001$.

[7] F. Constantinescu and E. Magyari. Problems in Quantum Mechanics. Pergamon, 1978.

[8] R. W. Robinet. Quantum and classical probability distributions for position and momentum. Am. J. Phys., 63(9) p. 823, 1995.

[9] H. N. Núñez-Yépez, C. A. Vargas, and A. L. Salas Brito. The one-dimensional hydrogen atom in momentum representation. Eur. J. Phys., 8(3) p. 189, 1987. 
[10] M. Abramowitz and I. A. Stegun. Handbook of Mathematical Functions (without Numerical Tables). NBS, 10 edition, 1972.

[11] I. I. Gớdman, V. D. Krivchenkov, B. T. Geilikman, E. Marquit, and E. Lepa. Problems in Quantum Mechanics. Addison-Wesley Pub. Co, authorized rev. edition, 1961.

[12] D. J. Griffiths. Introduction to Quantum Mechanics. Prentice Hall, 1st edition, 1995.

[13] L. D. Landau and E. M. Lifshitz. Quantum Mechanics: Non-Relativistic Theory, volume 3 of Course on Theoretical Physics, Vol 3. Pergamon Pr, 3 edition, 1981.
[14] Eugen Merzbacher. Quantum Mechanics. Wiley, 3 edition, 1997.

[15] I. S. Gradshteyn and I.M. Ryzhik. Table of Integrals, Series, and Products. Academic Press, 7th ed edition, 2007.

[16] M. S. Spiegel, S. Lipschutz, and J. Liu. Mathematical Handbook of Formulas and Tables. McGraw-Hill, 3 edition, 2009.

[17] Wolfram Research, Inc. Mathematica, Version 8.0. Champaign, IL, 2010. 